\title{
Aesthetic Value of Mirror Symmetry in Graphic Design
}

\author{
Ivan Budimir, Miroslav Mikota, Josipa Fotak, Hrvoje Grujić \\ University of Zagreb Faculty of Graphic Arts
}

\begin{abstract}
The primary goal of graphic design as a form of "commercial art" is to create a stronger aesthetic impression on graphic product users. In order to achieve better aesthetic quality, designers use mathematical compositional rules such as golden ratio or symmetry. This paper contains extensive discussion of the possibilities offered by different types of symmetry in graphic design. Moreover, in this paper we present the research results based on the method of experimental aesthetics and evaluated estimations of the survey participants on aesthetic value of the mirror symmetric form. For this purpose, four original test samples were designed, which were ranked by the respondents using the Liekert scale, according to their own aesthetic significance. Non-parametric Friedman's ANOVA and Wilcoxon tests were used to identify pairs of arithmetic means of ranks that statistically differ significantly $(\mathrm{p}<0.05)$. It was found that respondents considered that the highest quality test pattern was the one whose form was fully aligned with the mirror symmetry, thereby experimentally verifying the aesthetic value of the mirror symmetry.
\end{abstract}

Keywords: mirror symmetry, aesthetic value, graphic design, Friedman's ANOVA

\section{Introduction}

Creating design solutions for graphic products requires precise planning of its form in order to better reflect the elements the designer seeks to highlight. Although each design work is primarily dependent on the designer's creativity and experience, and realizes his ideas in an intuitive way, every designer is aware that high-quality design requires the use of geometric compositional rules which define the design form, like the golden ratio rule, rules of symmetry, asymmetry, stability, rhythm or optical balance [1]. The mentioned compositional rules closely link field of graphic design with the field of mathematics, whose geometric regularities represent an integral part of the graphic design [2]. Mathematical compositional rules are important in graphic design because they define the arrangement of all elements within the whole design and their mutual relationship with one another, thus securing aesthetic quality of design in order to achieve its full potential. It can be concluded that the original value and beauty of the design mostly depend on its form, which is designed on the basis of clearly defined mathematical compositional norms [3].

One of the definitions states that graphic design is a "commercial art" [4], which besides having artistic value, must also meet the demands of graphic product users. Therefore, graphic design should be adequately tested on survey participants to determine their aesthetic preferences, which is a research area of experimental psychology. More specifically, experimental 
aesthetics as a branch of experimental psychology tries to define the aesthetic value of the art work through psychological experiments [5]. For the purpose of testing the visual quality of compositions that are, to a greater or lesser extent, consistent with mirror symmetry, this paper uses the methodology of experimental aesthetics. The method is a combination of a visual experiment and a statistical test of the obtained results. In this paper, compositional design rules are interpreted mathematically, while their aesthetic value is evaluated experimentally. It is therefore evident that interdisciplinarity is a characteristic of a scientific approach used to carry out the discussion and set up the research presented in this paper (Figure 1).

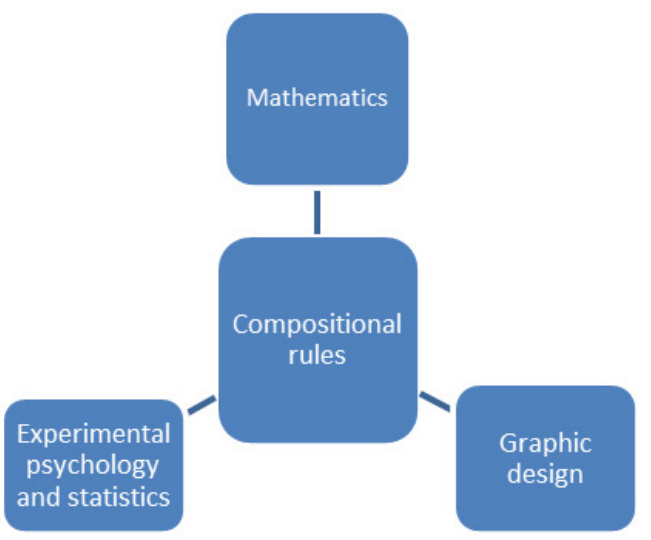

Figure 1. The paper is interdisciplinary related to graphic design, mathematics and experimental psychology

\subsection{Multicultural dimension of symmetry phenomena}

The phenomenon of symmetry is ubiquitous in nature starting from the structure of the smallest parts of the atom that are arranged symmetrically, to the regularities that are characteristic of the position of planets and stars and their regular layout within the huge galaxies in the universe [6]. Man experimentally observes symmetry at every step, in nature, in the structures of living creatures, objects surrounding it, in all areas of life, and thereby acquires a certain foreknowledge of symmetry. Symmetry spontaneously attracts human attention from the earliest pre-history of the human race to the present day. It has been shown that symmetry can be theoretically studied with many different aspects and can be applied in various ways in various fields. For example, symmetry is often expressed both in fine art and music art [7] but it is also possible to find examples of symmetry in literature [8]. The term symmetry is interpreted in philosophy [9], it is defined and analyzed in a number of natural science disciplines such as mathematics, physics, chemistry and biology [10]. In the mathematics, symmetries were researched and very precisely defined in the field of classical Euclidean geometry and the logical foundations, the axiomatics and the structure of symmetry were explored [11]. Discoveries within classical geometry associated with symmetries were extended and generalized in the 19th century in the framework of abstract algebra, where the symmetry is studied within the theory of Abel's groups that form a part of modern geometry known as algebraic geometry [12].

Since multiple manifestations of symmetry cannot be adequately described within one scientific area, many world class scientists are working on interdisciplinary unification of the results of various scientific disciplines in order to interpret the essential features of the mentioned phenomenon in new ways. It is therefore necessary to perform interdisciplinary, multicultural research of different variations of symmetry and related phenomena such as invariance, proportionality and rhythm, which are linking different fields of science, art and technology [13]. The symmetry research results pose great challenges to designers and artists, and can open entirely new possibilities in the field of graphic design, which is very demanding cultural venture.

Therefore, there is a need for researching of theoretical and formal hypothesis because of interdisciplinary linking of graphic design with mathematical sciences, especially with the field of Euclidean and algebraic geometry.

\subsection{Mathematical definition of mirror symmetry}

In mathematics, the concept of symmetry is very precisely defined within classical Euclidean geometry. The following text presents the precise logical definition of axial or mirror symmetry in the plane. Definitions of other types of symmetries are omitted (see [3], [14]). In order to precisely, axiomatically, define the concept of mirror plane symmetry, it is 
necessary to introduce the definition of plane isometry (def. 1, [14]).

Definition 1. Mapping $\mathrm{f}: \mathrm{M} \rightarrow \mathrm{M}$ is the plane isometry of $\mathrm{M}$ if it is

$$
d(f(A), f(B))=d(A, B) \text { for all } A, B \in M
$$

Now the concept of mirror symmetry can be axiomatically defined (def. 2. [14]).

Definition 2. For every line $\mathrm{p} \subseteq \mathrm{M}$ there is a unique isometry $\mathrm{s}_{\mathrm{p}}: \mathrm{M} \rightarrow \mathrm{M}$ different from $\mathrm{Id}_{\mathrm{M}}$ for which is $s_{p}(T)=T$ for every point $T \in p$. This isometry is called axial symmetry with respect to the line $\mathrm{p}$, and line $\mathrm{p}$ is called the axis of symmetry.

Taking into account the characteristic of the axial symmetry that it reflects points from one side of the line to the other side like a mirror, this type of symmetry is called mirror symmetry (Figure 2).

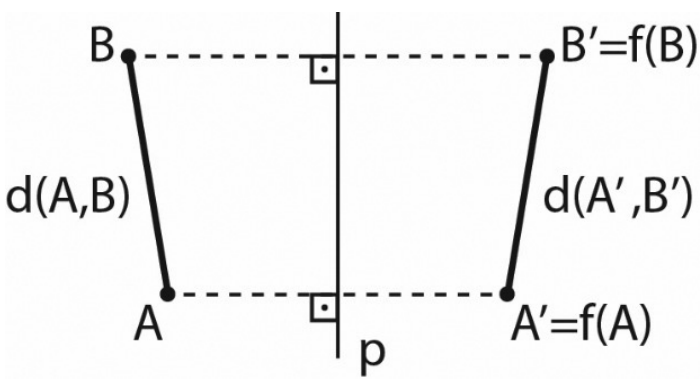

Figure 2. Mirror symmetry display

Furthermore, certain geometric figures in a two-dimensional space can be mirror symmetric (def. 3. [3]).

Definition 3. The geometric figure $\mathrm{F}$ is called a mirror symmetric with respect to the line $\mathrm{p}$ if for every point $T$ of this figure $(T \in F)$ there is a point $\mathrm{T}^{\prime}$ which also belongs to this figure $\left(\mathrm{T}^{\prime} \in \mathrm{F}\right)$ such that $s_{p}(T)=T^{\prime}$ (Figure 3 ). Accordingly $s_{p}$ is axial symmetry with respect to the line $\mathrm{P}$.

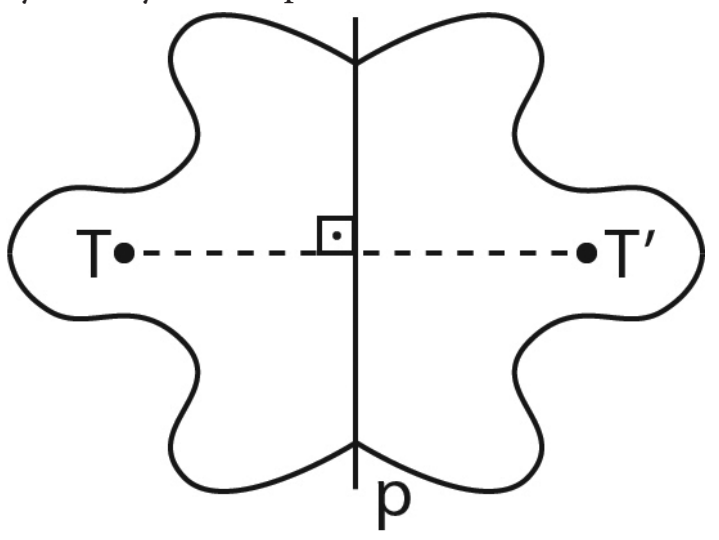

Figure 3. Display of the mirror symmetric figure
Mirrored symmetrical figures can be a very interesting graphic design motif.

\subsection{Overview of past research of aesthetic value of symmetry}

Everyday experience shows that the people's aesthetic attitudes are affected by symmetry, which manifests in various forms. In contemporary experimental psychology, numerous studies were being conducted in order to examine the impact of symmetry on human visual perception as well as on the formation of aesthetic stands.

For example, P. Locher has shown that symmetry and golden ratio used in visual forms and compositions affect visual discovery, focusing attention, and physiological excitement of respondents [15]. His flash experiments also confirm that symmetry is observed at first glance, so the eye attention is first focused on symmetry. The research of this author found that symmetry is also associated with the respondent's aesthetic attitudes. Also, the analysis of the eye fixation points shows that the axis of symmetry is used as a perceptual orientation where the eye attention of the examinees is focused. Locher's point of view, which the author cites in the conclusion of the above mentioned work, can be summarized in the following sentences: "The human nervous system that connects the eye and brain most likely functions on the principles of symmetry. The rapid and accurate detection of static symmetry by the perceptual system is most probably the first fundamental non-learned response to visual stimulation." [15] According to most perception theories that attempt to interpret the way the human visual system function, the origin used by the visual system is an axis of symmetry on which visual attention and analysis of visual content is anchored. Locher's results are of utmost importance to designers who seek to attract attention on the content of graphic design in order to use the design to communicate with the users as efficiently as possible.

Artists through all historical periods were always aware of high aesthetic value of symmetry. People appreciate symmetrical patterns that evoke feelings of harmony, order and subtlety of an artwork. In order to explain the reasons why people like symmetry, Reber et al. 
assumed that the aesthetic tendency to symmetry could be related to the way the human visual cognitive-perceptive system functions $[16,17]$. The hypothesis set by these scientists is based on the dynamics of information perceived by respondents. According to the mentioned authors, variables that influence the formation of aesthetic criteria are the beauty of the observed figures, the contrast between the figures and the background, repetition of stimuli and symmetries, and the prototypes that are characteristic for the particular culture to which the respondents belong. The same authors conclude that the experience of beauty is primarily based on the perceptive experiences of the respondents, which is only partially related to the characteristics of the perceived object. Furthermore, the same authors have concluded that symmetric objects are easier and faster to perceive than others because they contain a smaller amount of information than other forms. Similar results were obtained by Enquist and Arak who proved that symmetric patterns are perceived more efficiently than other objects [18]. Guidelines for graphic designers, which clearly follow the results of the above-mentioned scientific research, point to the advantages of using a compositional symmetry rule in order to create a design as attractive as possible.

Researches on aesthetic value of the form in which the symmetry is combined with the golden ratio [3] were also conducted. The symmetrical figure was moved diagonally in the field of view to determine whether the composition in which the position of the figure is in golden ratio is visually more acceptable than the others. The research results were negative. It was found that the aesthetic attitudes of the respondents were not affected by the position of symmetrical figures on the main diagonal. The authors concluded that the symmetry of the figure itself so strongly attracts the attention of the respondents, that they probably do not notice the position of figure. These results can be put in context with Locher's observations, according to which the symmetry is the convenient anchor point where the attention of the observer is focused, whose attention is so focused on the symmetry that their position is not relevant.
Researches in this area of experimental psychology have outstanding importance for graphic design, as they provide valuable information on responders' reactions to symmetri$\mathrm{cal}$ and other forms that can represent visible or even hidden part of graphic design. The aim of this paper is to discuss the possibility of applying symmetry in graphic design and to present the results of the evaluation of the visual quality of the mirror symmetry that was experimentally carried out by the method of experimental aesthetics.

\section{Experimental part}

The aesthetic value of the mirror symmetry was tested by the methodology of experimental aesthetics, which unlike philosophical aesthetics evaluates empirically the aesthetic experience that subjects have on visual forms [5], [19]. This chapter present the results of the visual research of symmetry, which occurred as part of the process of writing the final thesis at the Faculty of Graphic Arts, University of Zagreb [20]. The experiment that tested the aesthetic attitudes of subjects on mirror symmetry consisted of two parts:

a) Creation of specific original test samples, one of which was a sample with the elements that are aligned with the mirror symmetry, while the remaining three samples contained similar motives that deviate more or less from the mirror symmetry.

b) The visual experiment was carried out by a questionnaire in which the respondents were supposed to evaluate test samples using the Likert scale. Thus, In this way, we generated the data that the aesthetic value of the mirror symmetry was estimated using experimental aesthetic methods. All experimental results were treated statistically in STATISTICA 12 (StatSoft, Tulsa, USA).

\subsection{Design and making of test samples}

The experiment was carried out over the original test samples designed by the authors of this paper. Four different test samples were made. The samples were created by positioning yellow squares on the black base.

In that way four test samples were obtained, that is to say four test samples of a specific design. 
The principle of organization of elements in test samples for four cases is explained below.

Case 1 . The first test sample (Figure 4 ) is mirror-symmetric with respect to several axes of symmetry. When taking the imaginary axes of symmetry it is seen that they perfectly overlap the two sides in two directions, vertical and horizontal.

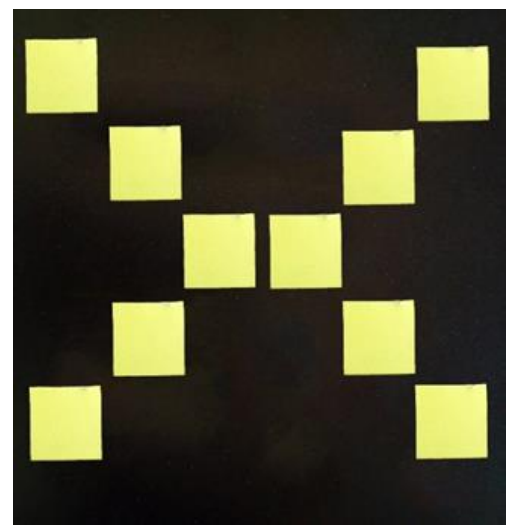

Figure 4. Test sample in which the elements are mirror symmetrical

Case 2. The second test sample (Figure 5) has little deviation from the mirror symmetry. Two yellow squares are displaced by 2 millimeters towards the bottom of the sample with respect to the previous sample (Figure 4), while other elements remain in their places.

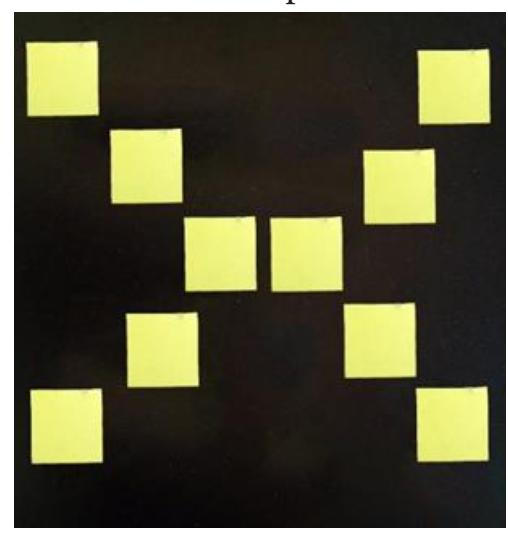

Figure 5. Test sample in which elements slightly deviate from the mirror symmetry

Case 3. The third test sample (Figure 6) has an obvious deviation from the mirror symmetry rule. In this case, more squares are shifted relative to the first case sample (Figure 4), which is completely mirror-symmetric.

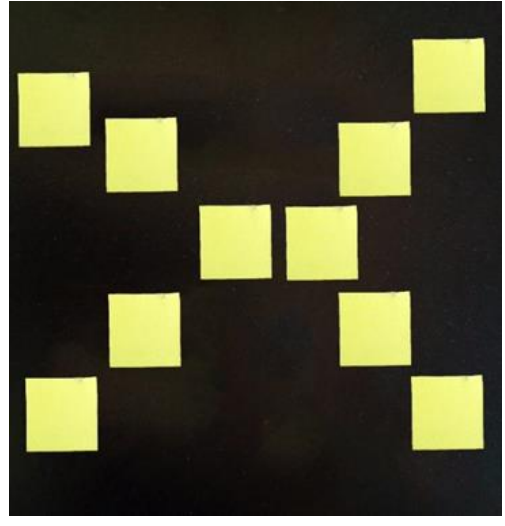

Figure 6. Test sample in which the elements are very different from the mirror symmetry

Case 4. The fourth test sample (Figure 7) is totally asymmetric and completely deviates from the mirror symmetry rule.

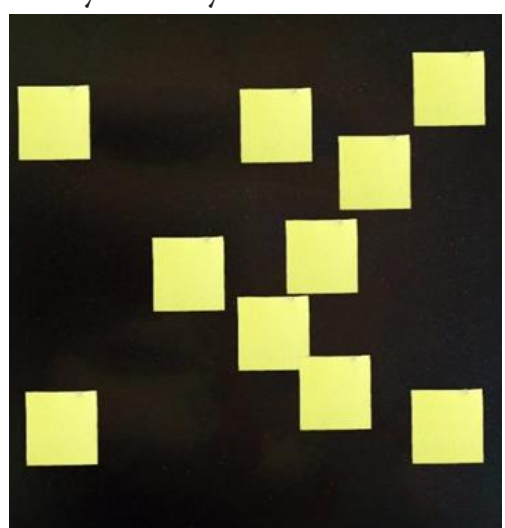

Figure 7. Sample in which the elements are asymmetric

\subsection{Visual experiment}

The visual experiment carried out was executed using a survey designed to test the visual quality of the mirror symmetrical shape of the graphic design [19]. Respondents were initially explained basic information about the survey itself and the reasons for conducting the survey. 52 respondents were interviewed. The survey examined the age of respondents and their social status, and found that all age groups were represented and that there were pupils, students, workers, unemployed and retired people among the examinees.

In the most important part of the survey, participants were given the task to rank test samples using the Likert scale, whereby the ratings should not be repeated. Ranks should have been assigned to their own sense of beauty of 
each individual respondent. The Likert scale was defined in the following way:

1 - I completely like it

2 - I like it

3 - I do not like it

4 - I totally do not like it

In this way, respondents had the opportunity to evaluate a particular test sample according to their own preferences. The survey was conducted online through the Google forms, which improved the implementation of the survey and simplified data collection. All data was sorted by Microsoft's Excel 2013 and were statistically analyzed.

\section{Research results and discussion}

In order to evaluate the visual quality of the mirror symmetry, descriptive statistical analysis of all the data collected by the experiment was performed using the STATISTICA 12 software package [21]. The aesthetic quality of each individual test sample (image)

$\overline{\mathrm{F}}_{\mathrm{i}} \mathrm{i}=1, \ldots, 4$ is defined by the arithmetic mean of all grades given by the subjects to the test sample. The rating given by $j$-th examinee $(j$ $=1, \ldots, 52)$ to the $\mathrm{i}$ - th sample $(\mathrm{i}=1, \ldots, 4)$ is marked with

$\mathrm{R}_{\mathrm{ij}}$. Expressed by the formula, the expression for the aesthetic value of the test sample is as follows:

$$
\overline{\mathrm{F}}_{\mathrm{i}}=\frac{\sum_{\mathrm{j}=1}^{52} \mathrm{R}_{\mathrm{i}_{\mathrm{j}}}}{52}, \mathrm{i}=1, \ldots, 4
$$

The arithmetic means $\overline{\mathrm{F}}_{\mathrm{i}}$ are presented in the second column of Table 1, which contains the corresponding standard deviations (

$\left.\overline{\mathrm{F}}_{\mathrm{i}}=\mu \pm \sigma\right)$. In addition, Table 1 also shows medians (Med), minima (Min), maxima (Max), and variance (Var) of test samples.

Table 1. Descriptive statistical analysis

\begin{tabular}{|c|c|c|c|c|c|}
\hline Sample & $\boldsymbol{\mu} \pm \boldsymbol{\sigma}$ & Med & Min & Max & Var \\
\hline $\mathrm{F}_{1}$ & $1,81 \pm 1,25$ & 1 & 1 & 4 & 1,57 \\
\hline $\mathrm{F}_{2}$ & $2,23 \pm 0,76$ & 2 & 1 & 4 & 0,57 \\
\hline $\mathrm{F}_{3}$ & $2,73 \pm 0,84$ & 3 & 1 & 4 & 0,71 \\
\hline $\mathrm{F}_{4}$ & $3,02 \pm 1,34$ & 4 & 1 & 4 & 1,78 \\
\hline
\end{tabular}

The presented results shows that the ranges between the minimum and the maximum in all test samples range from 1 to 4 , which is the minimum and maximum offered by the Likert scale. It is apparent that medians conform to the arithmetic means. Variance and standard deviations are relatively low, which means that the results obtained are of very high quality. The graphic display of the arithmetic means $\overline{\mathrm{F}}_{\mathrm{i}} \mathrm{i}=1, \ldots, 4$ of all the ranks assigned to each sample is shown in Figure 8.

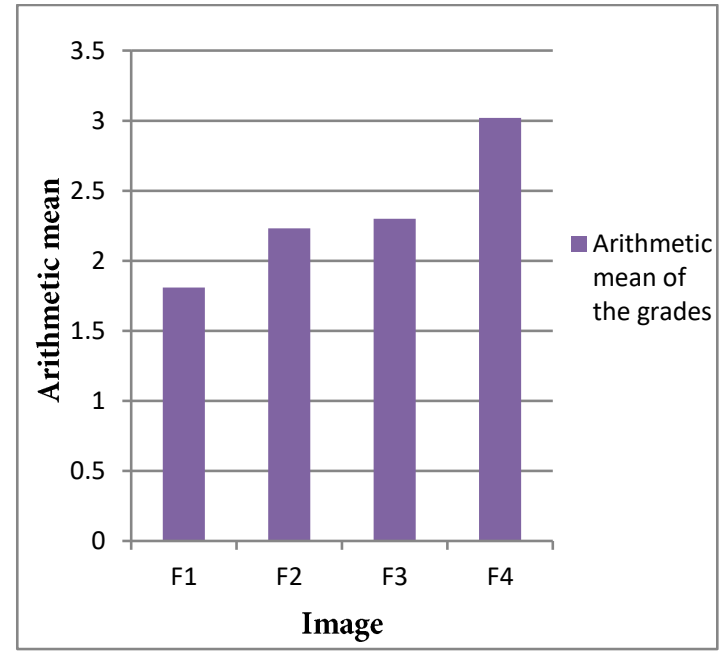

Figure 8. Graphic display of the arithmetic means

Furthermore, the compliance of all data with the law of normal distribution was tested using the Kolmogorov-Smirnov test (Table 2). Table 2 gives relevant statistical parameters such as Kolmogorov-Smirnov test statistics Max D and empirical p-values.

Table 2. Results of the Kolmogorov-Smirnov test

\begin{tabular}{|c|c|c|}
\hline Sample & Max D & p \\
\hline $\mathrm{F}_{1}$ & 0,39 & $\mathrm{p}<0,01$ \\
\hline $\mathrm{F}_{2}$ & 0,27 & $\mathrm{p}<0,01$ \\
\hline $\mathrm{F}_{3}$ & 0,3 & $\mathrm{p}<0,01$ \\
\hline $\mathrm{F}_{4}$ & 0,384 & $\mathrm{p}<0,01$ \\
\hline
\end{tabular}

It was found that all of the grades of any of the test samples do not follow a normal distribution (Table 2). The result obtained is expected because it is associated with the characteristics of the experiment performed in which the ranks were assigned.

Therefore, a non-parametric Friedman ANOVA analysis was performed for dependent samples with repeated measurements. The mentioned analysis tested the differences between Likert's arithmetic means for all 4 tested samples.

Statistical analysis has shown that Friedman's chisquare is ${K_{52}}^{2}=26,90$ and Friedman's statistical significance is $p=0.00001$. From these data it is clear that there are statistically significant differences between arithmetic means. Furthermore, a post hoc analysis was carried out, identifying test samples 
whose arithmetic meanings are statistically significantly different. Post hoc analysis was performed by Wilcoxon tests of comparison of sample pairs for case of dependent samples (Table 3). Table 3 itself contains the statistical test parameter, ie the T-value as well as the statistical significance of the test expressed by the p-value.

Table 3. The results of Wilcoxon tests

\begin{tabular}{|c|c|c|c|}
\hline Samples & $\mathrm{F}_{1}$ & $\mathbf{F}_{2}$ & $\mathbf{F}_{3}$ \\
\hline $\mathrm{F}_{2}$ & $\begin{array}{c}\mathrm{T}=304,00 \\
\mathrm{p}=0,015958\end{array}$ & - & - \\
\hline $\mathrm{F}_{3}$ & $\begin{array}{c}\mathrm{T}=321,00 \\
\mathrm{p}=0,003736\end{array}$ & $\begin{array}{c}\mathrm{T}=405,00 \\
\mathrm{p}=0,015591\end{array}$ & - \\
\hline $\mathrm{F}_{4}$ & $\begin{array}{c}\mathrm{T}=303,50 \\
\mathrm{p}=0,002114\end{array}$ & $\begin{array}{c}\mathrm{T}=338,00 \\
\mathrm{p}=0,003839\end{array}$ & $\begin{array}{c}\mathrm{T}=433,50 \\
\mathrm{p}=0,113050\end{array}$ \\
\hline
\end{tabular}

$\mathrm{P}$ values of virtually all pairs of arithmetic means are less than the limit value of 0,05 (Table 3 ) with the exception of the pair $\mathrm{F}_{3}$ and $\mathrm{F}_{4}$. This means that it can be concluded that there are statistically significant differences between all pairs of arithmetic means, which is not the case with the pair of arithmetic means of the $\mathrm{F}_{3}$ and $\mathrm{F}_{4}$ test samples, with a significance level of $\mathrm{p}<0.05$. There are no statistically significant differences for the arithmetic means of test samples $\mathrm{F}_{3}$ and $\mathrm{F}_{4}$.

Accordingly, the results of the study show that the best average of Liecert's ranks belong to the test sample $\mathrm{F}_{1}$, whose arithmetic mean of rank is

$\overline{\mathrm{F}}_{1}=1,81$. Its median is $\operatorname{Med}\left(\mathrm{F}_{1}\right)=1$, which is consistent with the arithmetic mean (Table 1). Also, given that its arithmetic mean is statistically significantly different from other arithmetic means $(p<0.05)$, it was clearly found to be the most preferred to the respondents (Table 3 ). This test sample is made exactly according to the mirror symmetry rule. Consequently, the respondents consider it aesthetically the highest quality test pattern, the shape of which is in accordance with the mirror symmetry. The next best ranked test sample is $\mathrm{F}_{2}$ whose arithmetic mean is $\bar{F}_{2}=2,23$, and median $\operatorname{Med}(F 2)=2$, which is in accordance with the arithmetic mean (Table 1). This arithmetic mean also statistically differs significantly from all other arithmetic means $(\mathrm{p}<0.05$, Table 3 ). This result also confirms the hypothesis according to which the aesthetic preferences of the subjects converge to the symmetrical form. Furthermore, test samples $\mathrm{F}_{3}$ and $\mathrm{F}_{4}$ are worst ranked and their arithmetic means do not differ significantly $(\mathrm{p}=0.113$, $\mathrm{p}$ $<0.05$, Table 3 ). The arithmetic mean of all the ranks obtained by the sample $\mathrm{F}_{3}$ is $\bar{F}_{3}=2,73$. The median of the $\mathrm{F}_{3}$ sample is $\mathrm{Med}(\mathrm{F} 3)=3$, which is expected taking the arithmetic mean into account (Table 1).
The pattern with an asymmetric shape was rated as visually the worst. Test sample $\mathrm{F}_{4}$ has an average of assigned ranks $\overline{\mathrm{F}}_{4}=3,02$ with median $\operatorname{Med}(\mathrm{F} 4)=4$ (Table 1). The subjects do not show affinity to the asymmetric form or to a shape that significantly deviates from the mirror symmetry.

\section{Conclusion}

Experimental testing by the method of experimental aesthetics that was carried out over 52 subjects of different sex, age and social status, confirmed the quality of graphic design form that is made according to the mirror symmetry rule. Precisely, four different test samples were tested, one of which is mirror-symmetric, the second deviates slightly, the third strongly deviates from the mirror symmetry, while the fourth test sample was completely asymmetric. The measure of aesthetic quality was the arithmetical mean of the ranks given to samples by all the respondents. The research results have shown that mirror symmetric form is of the best quality, and its aesthetic quality (arithmetic mean of all ranks) is statistically significantly different from the arithmetic mean of other test samples $(\mathrm{p}<0.05)$. That result was obtained using Friedman's ANOVA (

$\left.\aleph_{52}^{2}=26,90, p=0,00001\right)$ and Wilcoxon tests. The worst ranked was an asymmetric test sample, whose arrangement of elements was completely random and chaotic. The result obtained provides insight into the aesthetic preferences of graphic users considering the rules of the mirror symmetry, which show a tendency towards symmetrically designed shape.

The results obtained can be explained by the results of Locher's research, according to which symmetry strongly attracts the attention of the observer and thus affects the aesthetic attitudes of respondents [15]. Locher's explanation is related to the way of functioning of the human visual perception system that easily and quickly processes visual information from symmetrical forms. Moreover, mirror symmetry is present in the world around us which is also one of the reasons why people prefer symmetry. Therefore, the authors of this paper can strongly recommend to graphic designers the use of mathematical compositional rules of symmetry. 


\section{References}

1. Arntson, A. E. (2012). Graphic design basics. Wadsworth Cengage Learning. Boston.

2. Budimir, I., Mikota, M., Budimir, I. (2015). The aesthetic value of the golden ratio and rhythm of the photographs. Acta graphica. 26 (1-2). 46-52.

3. Budimir, I., Mikota, M., Budimir, I. (2015). Estetska vrijednost načela simetrije $u$ fotografiji u odnosu na pravilo zlatnog reza. Blaž Baromić 2015-Zbornik radova. Mikota, M. (ur.). Hrvatsko društvo grafičara. Zagreb. 90-107.

4. Reis, R. A. (2001). Careers in Art and Graphic Design. Barron's Educational Series. International Standard Book.

5. Locher, P. (2011). Contemporary experimental aesthetics: State of the art technology. I-perception. 2 (7). 697-707.

6. Darvas, G. (2007). Symmetry: Cultural-historical and Ontological Aspects of Science-Arts Relations; the Natural and Man-made World in an Interdisciplinary Approach. Springer Science \& Business Media. Basel-Boston-Berlin.

7. Yudo, L. H. (2017). A concert with symmetrical images. Symmetry: Culture and Science. 28 (1). 87-88.

8. Safuanov, I. (2014). Transformations of space and spatial forms in literature. Symmetry: Culture and Science. 25 (4). 319-322.

9. McManus, I. C. (2005). Symmetry and asymmetry in aesthetics and the arts. European Review. 13 (52). 157-180.

10. Kosso, P. (2000). The empirical status of symmetries in physics. British Journal for the Philosophy of Science. 51. 81-98.
11. Kinsey, L. C. \& Moore, T. E. (2002). Symmetry, Shape and Space: An Introduction to Mathematics Through Geometry. Springer Science \& Business Media. Emeryville.

12. Miles, R. (1988). Undergraduate Algebraic Geometry. Cambridge University Press. Cambridge.

13. Hostetler, S. C. Integrating Mathematics, Theory and Creativity: Visualizing Proportional Structures in Digital Media. Design Principles and Practices: An International Journal. 3 (3). 279-292.

14. Braić, S. (2012). Predavanja iz metodike nastave elementarne geometrije. Split.

15. Locher, P. (1989). The perceptual value of symmetry. Computers \& Mathematics with Applications. 17 (46). 475-484

16. Reber, R., Schwarz, N., Winkielman, P. (2004). Processing fluency and aesthetic pleasure: Is beauty in the perceiver's processing experience? Personality and Social Psychology Review. 8(4). 364-382.

17. Reber, R., Winkielman, P., Schwarz, N. (1998). Effects of perceptual fluency on affective judgements. Psychological Science. 9. 45-48.

18. Enquist, M. \& Arak, A. (1994). Symmetry, beauty and evolution. Nature. 372. 169-172.

19. Albert, W. \& Tullis, T. (2013). Measuring the User Experience: Collecting, Analyzing, and Presenting Usability Metrics. Elsevier Inc. Waltham. USA.

20. Fotak, J. (2016). Pravila simetrije u matematici i dizajnu. Završni rad. Mentor: Budimir, I. Sveučilište u Zagrebu Grafički fakultet. Zagreb.

21. Ross, M. S. (2009). Introduction to Probability and Statistics for Engineers and Scientists. Academic Press. International Edition. 